\title{
Ueber eine seltene Form von Atrophie der Haut.
}

\author{
Von \\ Hofrath Prof. Neumann in Wien. \\ (Hierzu Taf. XXVI u. XXVII.)
}

In der Literatur liegen über die idiopathische Atrophie der Haut nur einzelne casuistische Mittheilungen ror, wie von Buchwald, ${ }^{1}$ ) Touton ${ }^{2}$ ) und Pospelow. ${ }^{3}$ ) Kaposi ${ }^{4}$ ) gibt hierüber nur an, dass bei allen marantischen Personen, seltener bei in mittlerem Lebensalter stehenden eine Art diffuser, fortschreitender, idiopathischer Atrophie vorkomme, bedingt durch Schwund des subcutanen Gewebes, vielleicht anch durch eine gleichzeitige, der senilen ähnliche degenerative Veränderung der Cutis.

Einen Fall der in Rede stehenden Krankheit hat Kaposi in der Sitzung der dermatolog. Gesellschaft vom 24. März 1897 vorgestellt.

Ueber die im Alter stattfindenden eigenthümlichen Veränderungen der Haut habe ich ${ }^{5}$ ) bereits im Jahre 1861 berichtet. Es heisst daselbst:

„Die Cutis hat in ibrem Dickendurchmesser eingebüsst, was besonders an der Papillarschicht in eclatanter Weise hervortritt. An jenen Stellen nämlich, wo die Papillen auch im normalen Zustande klein sind (Stirn, Bauchwand), läuft die malpighische Schicht an alten Häuten in gleicher Flucht mit der geglätteten Lederhaut fort, und an solchen Orten, wo die Papillen im

1) Ein Fall von diffuser idiopathischer Hautatrophie. Areh. f. Derm. 1888, p. 554.

$\left.{ }^{2}\right)$ Ein F'all von erworbener idiopathischer Atrophie der Haut. Deutsche med. Wochenschrift 1886.

3) Idiopathische Atrophie der Hant. Ann. de Derm. et de Syph. 1886.

$\left.{ }^{4}\right)$ In seinem "Lehrbuch der Hautkrankheiten“ 1893, p. 711.

5) Sitzungsbericht der kaiserl. Akademie 1861. 
mittleren Lebensalter am mächtigsten entwickelt sind (wie an den Fingerspitzen), sind sie an der senilen Haut bedeutend rerkürzt und auch verdünnt. Diese Veränderungen kann man geradezu als eine Schrumpfung bezeichnen, welche nothwendigerweise auch ihren Einfluss in der Richtung der Adnexa nehmen muss. Das Gewebe einer derartig geschrumpften Cutis zeigt uns mannigfaltige Verunstaltungen, durch welche sich die retrograde Metamorphose bekundet."

Unna (in Orth's "Histopathologie der Hautkrankheiten“ 1894, p. 995) bemerkt, nachdem er die von mir beschriebenen Degenerationen der Cutis theils mit seinem Elacin, theils mit Collacin indentificirt:

"Was die übrigen von $\mathrm{Neumann}$ angegebenen Veränderungen betrifft, so kann ich die meisten Angaben bestätigen: die stärkere Pigmentirung und die Atrophie der Oberhant im Allgemeinen, die Verkürzung der Haarbälge und Erweiterung der Talgdrüsen, die Integrität eines auch die Follikel einhüllenden subepithelialen Bindegewebsstreifens, welcher die besprochenen Degenerationen nicht eingeht, die Erweiterung, resp. das Klaffen der meisten Capillaren, welches wohl auf eine grössere Starrheit des Bindegewebes, stellenweise auch auf eine leichte Verdickung der Wandung bezogen werden muss und das Vorkommen von zerstreuten Zellenherden."

Zu ähnlichen Erscheinungen können wohl prämatur pathologische Processe führen.

Die Zahl der klinischen Beobachtungen über diese Form von Atrophie der Haut ist jedoch noch viel zu klein, als dass es möglich wäre, ein zutreffendes Bild derselben zu geben, ebenso wenig liegen derzeit genügende histologische Untersuchungen vor.

Der Fall von Behrend ${ }^{1}$ ) diurfte, wie Caspary ${ }^{2}$ ) hervorhebt, eher als Ichthyosis aufzufassen sein. Bei dem bemerkenswerthen Falle von $\mathrm{Buchwald}$ geben weder die $\mathrm{Ab}$ bildung noch die äusserst kurze Beschreibung der mikroskopischen Befunde ein genügend klares Bild; ebenso wenig bietet Touton's Fall besondere Anhaltspunkte über die Natur dieser

3) Ein Fall von idiopathischer angeborener Hautatrophie. Berliner klin. Wochenschrift 1895, Nr. 6.

2) Ueber Ichthyosis foetalis. Vierteljahrschrift f. Derm. 1886. 
Affection, zumal eine histologische Untersuchung hier ïberhaupt nicht vorliegt.

Und doch ist die Kenntnis dieser Krankheit nicht nur wegen ihrer Seltenheit, sondern auch in diagnostischer Beziehung ron hohem Interesse, weil sie mit anderen Hautaffectionen, zumal der Pityriasis rubra und Ichthyosis simplex Aehnlichkeit zeigt.

Ich glaube daher zwei Fälle idiopathischer Atrophie der Haut, welche ich gegenwärtig in Beobachtung habe, mittheilen zu sollen. Vorerst mögen die bisher mitgetheilten Fälle des Vergleiches halber skizzirt werden.

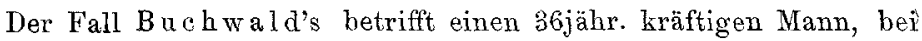
dem das Hautleiden im 20. Lebensjahre an den Knien begann, anfangs rapide Fortschritte machte, so dass es schon nach einem Jahre die volle stabile Ausdehnung erreichte. Ueber beiden Oberschenkeln, vorne jedoch das Schenkeldreieck freilassend und hinten his über die Glutaealgegend reichend, bildet die dünne, trockene, abschuppende Haut schräge und quere Faltungen. Sie lässt sich leicht verschieben und in grösseren Falten aufheben, welche nur langsam wieder verstreichen. Durch diese atrophische Haut, welche sich meist scharf gegen die gesunde Haut abgrenzt, schimmern deutlich die darunter liegenden Gefässe durch, namentlich wenn beim Stehen des Pat. sich die Venen praller füllen; dabei nimmt auch die vorher blassbraune Haut ein mehr cyanotisches Colorit an. Die atrophische Haut ist nur äusserst sparsam mit dünnen weichen Härchen besetzt, grosse Fläehen sină völlig haarlos.

Sowohl bei spontaner Schweissbildung wie nach subcutaner Injection von Pilocarpin bleiben die atrophischen Partien fast trocken, während die gesunden Stellen eine lebhafte Schweisssecretion zeigen.

Keine Sensibilitätsstörung, Tastsinn und Temperaturwahrnehmung normal. Die Nuskeln unter den erkrankten Hautstellen waren schwäeher als bei dem sonst robusten Körperbau zu erwarten war. Elektrische Erregbarkeit normal.

Der Maun hatte von seiner Hautkrankheit keinerlei Unbequemlichkeiten und über keinerlei Störungen des Gesammtbefindens zu klagen.

Die histologische Untersuchung ergab vollkommenes Fehlen des Fettgewebes, Gesammtatrophie, auffallende Sparsamkeit der Schweissdrüsen und Haarbälge, sowie Fehlen der Papillen. Auffallend erschien die Beschaffenheit des Bindegewebes dicht unter der (iibrigens glatt verlaufenden) Epithelgrenze, welches wie gequollen aussah, ausserdem mit reichlichen Zellkernen durchsetzt war. Letztere waren zum Theil um Gefässe, Drüsen und Haarbälge, theils isolirt und unabhängig von diesen in Gruppen angehäuft, Gefässe und Nerven normal.

2. T'outon. Bei einem 57jährigen, starken und corpulenten Mann begann die Affection vor 35 Jahren an beiden Händen und Füssen und 
breitete sich langsam von der Peripherie der Extremitäten nach oben auf beiden Seiten meist symmetrisch aus. Im Anfang verlief der Process viel rascher, später scheint eine bedeutende Verlangsamung, wenn nicht gar ein Stillstand in demselben eingetreten zir sein.

Am stärksten ist die Dorsalfläche der Hände afficirt; es macht den Eindruck, als ob die Hand in einem zu weiten, schlottrigen Handschuh steckte. Die Haut in Runzeln und Falten gelegt, die von diesen umschlossenen Felder sind feiner gerunzelt, glanzlos, trocken. Durch die diffus tief dunkelroth gefärbte Hant schimmert ein reichliches Venennetz durch, als ob es unmittelbar unter der Oberfläche läge; grössere Venen und die Sehnen wölben die Hautdecke vor. Diese ist leicht verschieblich, stellenweise papierdünn, was besonders beim Erheben einer Falte auffällt, die dann nur allmälig wieder sich ausgleicht. Die Nägel sind längsriefig. Auch an der Vola manus über Daumenballen und Kleinfingerballen sind äbnliche aber geringere Veränderungen. Die feinen Linien und Furchen des Handtellers, welche der Anordnung der Papillen entsprechen, fehlen hier. Am Vorderarm zieht sich die Affection in Form eines schmalen Streifens an der Streckseite bis über das Ellenbogengelenk hinauf. Die Grenzen der erkrankten Haut gegen die gesunde sind ziemlich scbarf, so jedoch, dass die Randpartien weniger intensiv ergriffen (also dicker und blässer) sind. Die unteren Extremitäten zeigen eine ähnliche aber weniger ausgedebnte Erkrankung, die nicht über das Kniegelenk hinaufreicht. Am Unterschenkel, wo der Process am wenigsten weit vorgeschritten ist, ist die Haut gespannt, pergamentartig weiss, glänzend und stellenweise mit reichlichen dünnen rundlichen oder polygonalen Schuppen bedeckt. Ueber allen erkrankten Partien finden sich zerstreut kurze Lanugohärchen. Fs besteht Hyperidrosis pedum.

Sensibilität und Motilität intact, ebenso Muskelkraft. Die atrophischen Stellen an Händen und F'üssen bei stärkerem Druck empfindlich.

Pospelow beschreibt zwei Fälle von „idiopathischer Atrophie der Hant". In beiden Fällen kam die Erkrankung bei 50jähr. Frauen zur Beobachtung.

Im ersten Falle ist die Haut der 1. oberen Extremität vom Handrücken bis zur Schulter und an der 1. Unterextremität vom untern Drittel des Oberschenkels bis zur Fusssohle afficirt. Nach Angabe der Pat. hat das Leiden in ihrem 17. Lebensjahre nach einem längeren Marseh in Schnee und Kälte mit rothen Flecken am l. Oberarm begonnen, die nach Ablauf eines Jahres eine Verdünnung der Haut zur Folge hatten. Die untere Extremität erkrankte 27 Jabre später als der Arm.

Die Haut ist an den afficirten Stellen faltig, trocken, ziemlicb kühl und livid verfärbt. Die Epidermis glänzt, ist schuppig und trocken. In Folge der zahlreichen Falten sieht die Haut ähnlich wie zerknittertes Cigarettenpapier aus. Ueber der Ferse lässt sich die Haut wie von einem gebratenen Apfel kegelförmig abheben. Durch die verdünnte Haut schimmern die Gefässe durch. Das subcutane Fett fast völlig ge- 
schwunden, auch die darunter liegenden Muskeln sind schwächer entwickelt. Durch Aetherspray lässt sich keine Gänsehaut erzeugen. Haarfollikel sind kaum nachweisbar. Keine Sensibilitätsstörung. Bei Zimmertemperatur ist der kranke Arm gegen Kälte mehr empfindlich als andere Körpertheile. Sebumsecretion gering, Schweisssecretion dagegen soll normal sein. Aus den histologisch untersuchten Hautstückchen geht hervor, dass die Atrophie nicht alle Hautschichten in gleicher Weise betrifft. Am auffallendsten schien der Schwund der Muskeln. Die ,horizontale Muskelschicht" ist theils mit Rundzellen infiltrirt und verdickt, theils so bedeutend atrophirt, dass man an ihrer Stelle nur geschrumpfte Bindegewebsbündel findet. Die Arrectores pilorum bestehen meist nur andeutungsweise. Aus diesen Veränderungen erklärt sich die Unfähigkeit der Haut, eine Gänsehaut zu bilden. Stratum corneum stellenweise verdickt, strecken weiseverdünnt. O eh l'sche u. Langerhans'sche Schicht sind verdünnt. Stratum Malpighii zeigt eine Abflachung der Papillen und leichte Erweiterung der Capillarschlingen. Talgdrüsen "ein wenig" atrophisch. Schweissärüsen haben fast gerade, kaum merklich spiralige Ausführungsgänge, während die Drüsenknäuel mehr flach horizontal liegen.

Beim zweiten Fall Pospelows begann die Erkrankung erst vor zwei Jahren, indem auf der Dorsalfäcbe des rechten Handgelenkes zwei dunkelrothe Flecke sich bildeten. Gegenwärtig ist sie am stärksten am Vorderarm, so dass die Sehnen durchschimmern und die "feinen Anastomosen der Nerven" deutlich sichtbar wurden. Je höher hinauf zur Schulter, desto weniger intensiv war die Veränderung und bestand in der Claviculargrube nur mebr inselförmig. In ähnlicher Weise, aber in bedeutend schwächerem Grade waren die 1. obere Extremität und beide untere Fxtremitäten bis ad nates afficirt. "Schweissdrüsenabsonderung ist etwas erhöht, die Talgabsonderung dagegen - wie es scheint - vermindert.“

Jonathan Hutchinson mag wohl, wie Behrend erwähnt, äbnliche Fälle beobachtet haben, und wäre vielleicht ein Fall, den Hutchinson') beschreibt, hieher zu rechnen. Bei dem 61jähr. Pat. waren nur Gesicht, Hände nnd Füsse frei, ebenso Scrotum. Sonst war die überall mit Schuppen bedeckte Haut ähnlich wie verknittertes Papier.

Ein derzeit a $\mathrm{n}$ meiner Klinik in Behandlung stehender $32 \mathrm{jähr}$. Taglöhner aus Mähren gibt an, dass sein Leiden vor zwei Jahren mit Röthung and schuppenbildung an den Unterschenkeln begonnen habe, ein Jahr später an Brust und Oberarm.

Von Juli bis September 1896 stand er dureh 12 Wocken an der Klinik Kaposi, später in ambulatorischer Behandlung von Doc. Dr. Ehrmann, am 31 . October 1696 wurde derselbe an meiner Klinik mit folgendem Status praesens aufgenommen:

Der Kranke ist gross, mässig genährt, hat ausser leichtem Frösteln geringem Jucken und allgemeiner Mattigkeit keine ernsten Beschwerden.

1) The Lancet $1875 \mathrm{I}$. 
Die Hautaffection ist über den grössten Theil der Körperoberfäche verbreitet, besonders intensiv am Stamm, den Oberarmen und Unterextremitäten, nur Gesicht-, Hals- und Scrotalhaut frei.

An den seitlichen Thoraxpartien in symmetrischer Weise vom Rippenbogen bis zur Brustwarze und gegen die Axilla hin, und in ganz gleicher Weise am Rücken bis zu den Nates, die Hut diffus geröthet, an einzelnen Stellen bräunlichroth, an anderen mehr livid, allenthalben gerunzelt, "zerknittertem Cigarettenpapier" vergleichbar, merklich verdünnt und atrophisch. Ueberall besteht Abschilferung der Epidermis, theils fein kleienförmig, theils in Form grösserer, dünner, mattweisser oder etwas glänzender, durchsichtiger Schüppchen; Ietztere sind fleckweis in etwas vertiefte Hautfelder eingelagert, die nach Ablösung der Schuppen als dunkelrothe, mit dünner, runzliger Epidermisdecke versehene Stellen erscheinen. Die meisten Schuppen adhäriren ziemlich fest. Haare fehlen fast vollständig, nur an den weniger intensiv ergriffenen Partien finden sich spärliche Lanugohärchen. An der Bauchwand ist die Röthung und Dünne der Haut weniger beträchtlich; hier finden sich zumeist linsengrosse, ganz weisse Flecken, welche an der Peripherie durch einen pigmentirten Saum begrenzt sind; sie liegen auch etwas tiefer, die Haut erscheint hier eingesunken.

Am weitesten gediehen ist die Erkrankung an der Hinterfäche des linken Oberschenkels; hier ist die Haut dunkel rothbraun gefärbt, stark runzlig, fast in Falten gelegt. Eine aufgehobene Hautfalte gleicht sich nur langsam wieder aus.

Die innere Schenkelfäche ist viel weniger ergriffen, hier ist die Haut namentlich gegen das Genitale hin noch ziemlich glatt. Die Venen an der Beugeseite nicht unbeträchtlich dilatirt, ihre Transparenz durch die verdünnte Haut erhöht. Die Kniekehlen sind frei.

An den Unterschenkeln finden sich analoge Veränderungen in Form umschriebener chagrinirt aussehender Plaques.

An den peripheren Partien der veränderten Hautstellen ist die Färbung blassroth und über diese hinaus noch abnorm trocken, runzlig und abschilfernd.

An der linken Seite der Bauchwand, an den Oberschenkeln, am Hals und Nacken und besonders um den Ellbogenhöcker, aber auch sonst an der Hautoberfläche zerstreut, finden sich zahlreiche blassbraune oder dunkelbraune, stecknadelkopfgrosse Epheliden und Pigmentflecke, welche an den weniger afficirten, mehr weissen Hautpartien stärker hervortreten. Sie sind auch am Gesicht, wie Nase und Wangen vorhanden. Mundschleimhaut intact, ebenso Finger- und Zehennägel. Sämmtliche tastbaren Lymphdrüsen leicht vergrössert.

16. Februar 1896. Nach $3 \frac{1}{2}$ monatlichem Spitalsaufenthalte und Gebrauch von über 400 asiatischen Pillen erscheint die ursprüngliche dunkle Röthung nicht mehr so auffällig, die Haut mehr braun gefärbt, die Schuppen zum Theile abgestossen; namentlich am Rücken einzelne Leisten etwas flacher, mehr wellenförmig verlaufend. Zwischen den 
Schulterblättern haben sich einzelne weisse Stellen, die unter dem Hautniveau liegen, entwickelt, gleich den an der Bauchwand bereits bestandenen. An den Nates und Oberschenkeln ist jetzt die Affection stärker, so dass beträchtlich hohe leisten hervortreten, welche in den von ihnen gebildeten Furchen schmutzig grünliche Schuppenmassen einschliessen.

An der Bauchwand dichtgedrängte linsengrosse weisse Flecke mit peripherer Pigmentirung, an der Brust dunkler gefärbte Pigmentflecke. Auch am Halse scheinen die Pigmentflecke und Epheliden etwas vermehrt.

An den Oberarmen und über der Schulter ist die Haut stark gerunzelt, polygonal gefeldert und von queren Riefen durchzogen.

Hände, Genitale und Mleocruralfurchen frei. - Die Schweissabsonderung erfolgt bloss am Genitale, am Gesichte, an den Händen, in der Achselhöhle und im Schenkeldreieck, was auch durch Pilocarpin-Injectionen constatirt werden konnte.

Sensibilität intact. Das Körpergewicht hat um 3 Kilogramm abgenommen. Doch gibt der Kranke an, sich subjectiv besser zu befinden; Jucken und Frösteln sind verringert. Harn frei von Eiweiss und Zucker.

Im November 1896 stellte sich der 23 Jahre alte Schlosser J. R. vor, welcher nach seiner Angabe bis auf Kinderkrankheiten sonst immer gesund war. Die Hautaffection besteht seit 10 Jahren und soll im Anschluss an Scharlach entstanden sein.

Der Kranke isti mässig genährt, Haut und Schleimhäute sind blass. Es finden sich an der Körperoberfläche, Gesichts- und Kopfhaut, Handund Fussrücken ausgenommen, zumeist thaler- und flachhandgrosse, an den Unterschenkeln über weit grösseren Strecken ausgebreitete Hautveränderungen. Diese variiren in ihrem Aussehen an verschiedenen Stellen in erheblicher Weise. An der Vorder- und Seitenwand des 'Thorax finden sich zumeist abgeflachte punkt- bis schrotkorngrosse, braunrolhgefärbte im Centrum von punktförmig erweiterten Gefässen durchzogene Efflorescenzen, welche an der Oberfläche mit fest anbaftender Epidermis bedeckt sind. An einzelnen Stellen, zumal der Bauchwand und Inguinalgegend sind diese Eff lorescenzen bereits resorbirt, an ihrer Stelle findet sich eine Felderung der Haut in der Art, dass im Centrum die Stellen etwas eingesunken und an der Peripherie durch Leisten begrenzt sind. An anderen Partien, insbesondere dem Rücken gleichfalls thalergrosse Stellen, welche abgeflacht, theils blassroth, theils rostbraun gefärbt sind; an diesen keine Felderung. An den kranken Partien ist der Haarwuchs ein ganz normaler, die Schweiss-Secretion fehlt jedoch gänzlich. Subjective Erscheinungen nicht vorhanden. Im Harn kein Eiweiss.

In diesem Falle lässt sich die Entwicklung der Hautaffection in ihren verschiedenen Stadien ziemlich gut studiren. Anfangs erscheinen punktförmige, von erweiterten Gefässen durchzogene Knötchen, welche gegen die Peripherie hin wachsen und die Grösse eines Schrotkornes erreichen, anfangs mehr hellroth gefärbt sind, und je älter, desto mehr in braun übergehen. In weiterem Verlauf tritt Abflachung und 
dementsprechend Hervortreten der Leisten ein. Später kommt es zur Resorption der Knötchen u. zw. noch bevor dieselben einen grösseren Umfang erreicht haben, die betreffende Partie erscheint flach, hellroth bis braunroth gefärbt; schliesslich entstehen, wie z. B. an der Bauchwand, weisse vitiligoartige Stellen, welche eingesunken und an der Peripherie von einem braunrothen Rand begrenzt sind.

Die mikroskopische Untersuchung wurde in beiden Fällen vorgenommen.

Schon beim Excidiren der Hautstückchen, insbesondere aus einer weiter vorgeschrittenen Partie des ersten Falles, bietet sich dem Messer ein beträchtlicher Widerstand dar, als würde man Narbengewebe durchtrennen. Die Schnittfläche erscheint weissglänzend.

Die zu untersuchenden Hautstückchen wurden theils in eine Mischung von 2 Th. Müller'sche Flüssigkeit und 1 Theil $10 \%$ Formalinlösung gebracht, theils sofort in Alkohol gehärtet. Ein Theil wurde in zusammenhängender Serie geschnitten.

Als Färbemethoden kamen zur Anwendung:

1. Hämalaun-Eosin.

2. Färbung nach Van Gieson (Sehnitt wird in Hämatoxylin Grübler wäbrend 1/a Stunde überfärbt; nach gründlichem Auswaschen Contrastfärbung und damit gleichzeitig Differenzirung in einer Mischung von Pikrinsäure (wässerig conc.) und Säure-Fuchsin (conc.) von letzterer wird einem Uhrschälchen mit Prikrinsäure tropfenweise so viel zugesetzt, dass man durch dasselbe hindurch Schriftzïge auf einem untergelegten Blatt Papier noch deutlich erkennen kann).

3. Färbung der elastischen Fasern mit saurem Orcein nach Unna-Tänzer.

(Schnitt bleibt darin für eine Stunde im Brutofen, wird hierauf in $1 \%$ Salzsäure-Alkohol differenzirt.) Vorgefärbt wurde mit Lithion-Carmin.

4. Weigert'sche Färbung.

5. Mastzellenfärbung mit polychromem Methylenblau (Schnitt darin 1/4 Stunde überfärbt, nachträglich durch Glycerinäthermischung entfärbt).

6. Lithioncarminfärbung für das Pigment.

7. Auf Eisengehalt des Pigments wurde mit Ferrocyankaliumlösung und Salzsäure geprüft. 
8. Für die Prüfung auf Amyloid wurde die Jodreaction und Methylviolettreaction verwendet.

9. Für Collacin und Elacin nach Unna: Polychromes Methylenblau 10 Min., differenzirt in $33 \%$ wässriger Tanninlösung; rorgefärlot in kalter saurer Orceinlösung, Contrastfärbung des Collagengrundes mit S. Fuchsin.

10. Endlich wurden mehrere Schnitte noch mit verschiedenen, anderen Farbstoffen gefärbt, so mit Thionin, Toluidinblau etc.

An den Schnittpräparaten, welche mit verschiedenen Färbemethoden zum grossen Theil von dem Arzte meiner Klinik Dr. Matzenauer hergestellt wurden, konnte ich folgende pathologische Veränderungen constatiren (Taf. XXVI und XXVII.):

Die verhornten Epidermislagen sind in Form von unregelmässigen, zumeist aus kernlosen Zellen bestehenden bandartigen Streifen von der übrigen Epidermis abgehoben. Diese ist in ihrer Gesammtheit verdünnt. Während die Körnchenzellenschicht auf einen ganz dïnnen Streifen reducirt ist und vielfach fehlt, erscheinen die Stacheln und Riffe der folgenden Zellenlagen höchst auffallend grösser.

Die Epidermis verläuft an den meisten Stelien, da der Papillarkörper zum grossen Theil völlig abgeflacht ist, in gleicher Flucht mit dem Cutisgewebe.

Dieses bietet in seinen verschiedenen Lagen ein wesentlich verschiedenes Bild. Während dasselbe in den oberen Lagen mehr netzförmig angeordnete Bündel zeigt, die einzelnen Bindegewebs-Fibrillen schmäler sind und weniger intensiv gefärbt erscheinen, bilden die tiefen Lagen oft breite massige und stärker tingirte Züge, welche meist horizontal und wellenförmig verlaufen.

An einem nach Van Gieson gefärbten Präparate tritt die verschiedene Farbennuancirung der oberen und tieferen Cutislagen besonders deutlich hervor, speciell bei Tageslicht. Während erstere eine zarte und vollkommen diaphane pfirsichblütenrothe Farbe angenommen haben, erscheinen letztere in mehr aufdringlicher, oft leuchtend gelbrother Feuerfarbe; sie nehmen dabei ein mehr opakes aber homogenes Aussehen an. Und während die obere Cutislage sich nur auf eine dünne subpapilläre Schichte beschränkt, betragen die in ihrer Form 
und Farbe verschieden sich darstellenden Bindegewebsbündel der folgenden Cutislage die Hauptmasse der gesammten Cutis.

Wo jedoch in diesen tieferen Cutislagen Schweissdrüsenknäuel, Gefässe und Nerven liegen, sind diese von einem zarten Bindegewebsfasernetz umsponnen, welches durch seine schöne rosarothe Farbe und zartere Form sich scharf von dem mehr bomogenen Aussehen der übrigen dichten, massigen Bündel unterscheidet.

Weiters finden sich in den oberen Lagen Rundzellenwucherungen, jedoch nicht ausschliesslich um die Gefässe, sondern in Form von Nestern im Cutisgewebe aggregirt und greifen an mehreren Stellen auch in das Rete iiber. Entsprechend den Stellen, wo die Cutis von Rundzellenwucherung durchsetzt erscheint, ist die Hautoberfläche leistenförmig vorgewölbt, die Papillen noch stellenweise erhalten. Dagegen entsprechen den bereits narbig atrophischen Cutispartien Einsenkungen der Hautoberfläche, und hier fehlen Papillen gänzlich, das Rete ist verdïnnt, die Hornschichte dariiber dicker und in Fetzen abgehoben.

Die tieferen Lagen der Cutis sind nur an den Gefässen mit einer geringen Menge von Zellwucherungen durchsetzt.

Blut- und Lymphgefässe finden sich in reichlicher Menge in der Umgebung der Zellinfiltrate in der oberen Cutislage. Die Arterien sind stellenweise mit Wucherungen an der Adventitia versehen; hier finden sich Venen mit klaffendem Lumen und mit Blutkörperchen strotzend erfüllt; die Lymphgefässe sind oft deutlich mit einer einfachen Endothellage bekleidet. Nerven sieht man in den tieferen Cutislagen und Subcutis manchmal in langgeschlängeltem Verlauf.

Der Panniculus adiposus ist wohl zum grossen Theil geschwunden, jedoch an einzelnen Präparaten noch theilweise erhalten. Was besonders auffällt, ist die Rareficirung der Adnexe. Allerdings finden sich Schweissdrüsenknäuel häufig genug, intacte Ausführungsgänge sind jedoch nirgends zu sehen. Desgleichen sind die Haarbälge rareficirt, womit der klinische Befund in Einklang steht, indem die erkrankten Stellen ganz haarlos sind. Die Zellen der inneren und äusseren Wurzelscheide zeigen entsprechend den Arrectores pilorum 
kolbenartige Fortsätze. Es entsteht dadurch ein Bild ähnlich einer acinösen Drüse. Die Ausbuchtungen entstehen wahrscheinlich durch Contraction der Arrectoren. Diese sind vielfach hypertrophisch und finden sich auch an solchen Stellen, wo in den folgenden Schnitten der Serie weder ein Haar noch eine Talgdrüse aufgefunden werden konnte.

Talgdrüsen wurden überhaupt in keinem der Schnitte angetroffen. An ihrer Stelle liegt häufig ein mehr oder weniger straffes oder lockeres Bindegewebe, welches gemeinsam mit dem eigentlichen Haarbalg das Haar dicht umscheidet.

Weiters finden sich noch folgende Veränderungen:

Die in den oberen Lagen dichtgedrängt angehäuften Rundzellen sind zum Theil mit diffusem Pigment erfüllt, ausserdem sind noch zahlreiche Chromatophoren, zumal an jenen Stellen, woselbst das Pigment in der Epidermis fehlt. Einzelne Chromatophoren haben noch jene filamentären Fortsätze, welche für die Fortschaffung des Pigments aus der Epidermis dienen (Ehrmann). Nur sporadisch liegt das Pigment frei in der Cutis, während die Chromatophoren schon geschwunden sind. An jenen Stellen, wo Pigment in der Epidermis liegt, sind die Chromatophoren im Cutisgewebe nur in geringer Zahl vorhanden. Wo jedoch die Epidermis pigmentlos ist, findet sich in der Tiefe eine grössere Menge von Chromatophoren ror. Das Pigment erwies sich durchaus als nicht hämosiderinhältig, indem die Probe mit Ferrocyankaliumlösung und Salzsäure negativ ausfiel.

Die Färbung anf elastische Fasern mit saurem Orcein (nach Unna-Tänzer) zeigt ein reichliches, dicht verzweigtes und verfilztes Netzwerk ron elastischen Fasern. Wo noch Papillen rorhanden sind, lassen sich elastische Fasern stellenweise bis dicht an die Epidermislagen verfolgen. Wo der Papillarkörper jedoch verstrichen ist, fehlen sie ebenso wie in den mit Rundzellen dicht infiltrirten Gewebspartien. Am reichlichsten finden sie sich an jener Zone, wo die oberflächliche und tiefere Cutislage aneinander grenzen, während sie in der letzteren selbst wieder in geringerer Menge auftreten.

Mastzellen zeigen sich bei Färbung mit polychromem Methylenblau und nachträglicher Differenzirung mit Glycerinäthermischung ziemlich zahlreich in den kleinzellig infiltrirten 
Cutispartien der oberflächlichen Lagen und ebenso an jenen Stellen der tieferen Cutisschicht, wọ Blut- und Lymphgefässe, sowie Schweissdrüsenknäuel liegen.

Diese sind hier ganz umsponnen von den langen Ausläufern der Mastzellen, die oft in fadenförmigen Fortsätzen sich deutlich durch die Lymphspalten durchzwängen. Sonst trifft man sie zwischen den tiefer gelegenen Cutisbündeln nur ganz vereinzelt und immer deutlich in Lymphspalten liegend als langgestreckte Züge von Haufen feurig rothgefärbter Mastzellenkörnchen. In den oberflächlichen, bereits atrophischen Cutispartien sind sie gleichfalls nur in geringer Zahl vorhanden.

Die vom zweiten Fall excidirten Hautstiickchen zeigen den Process in einem weit jüngeren Stadium.

Der Papillarkörper ist überall noch grösstentheils wohl erhalten, nur wenig abgeflacht, die Epidermisdecke in ihrer Gesammtheit etwas rerdünnt. Die Hornschichte in bandartigen Streifen abgehoben. Die Körnchenzellenschichte fehlt meist vollständig, oder, wo sie vorhanden, ist sie es nur als ganz dünner Saum. Die Stachelzellenschichte ist bedeutend geringer entwickelt im Vergleich zu den Präparaten des ersten Falles. Degenerative Veränderungen der Cutis sind hier nicht nachweisbar. Eine Differenz in der Färbung und Configuration der oberen und tieferen Cutislagen ist gleichfalls nicht vorhanden.

Dagegen finden sich reichlich Zellwucherungen zumeist in herdweiser Anordnung dicht unter den Epidermislagen, an welchen häufig genug die Basalzellen fehlen, so dass das kleinzellige Infiltrat in das Rete Malpighii unmittelbar iibergeht, resp. in dasselbe einbricht. Es sind kleine Zellen mit kleinem, stark tingiblem Kern, der theils kuglig oder oval geformt ist, theils durch seine länglich-spindelförmige Gestalt sich als Abkömmling der fixen Bindegewebszellen documentirt, deren Kerne vermehrt sind. Mastzellen finden sich ziemlich reichlich, Plasmazellen nicht.

Diese Zellanläufungen beschränken sich zumeist auf den Papillarkörper und das Stratum subpapillare; die tieferen Cutislagen sind grösstentheils intact, und nur ab und zu setzt sich das Infiltrat in Form eines schmalen, nahezu geradlinig verlaufenden Streifens zwischen den Cutisbündein nach der 
Tiefe fort, wo es manchmal wieder in einem kleineren Infiltratherde endet. Intacte Schweissdrüsen-Ausführungsgänge sind nirgends zu sehen, ebensowenig Talgdrüsen. Auch die Arrectores pilorum erscheinen rareficirt.

Die Gefässe sind in ihrer Wandung stellenweise verdickt und häufig von Zellwucherung begleitet.

Ueberblicken wir das Ergebniss der klinischen und histologischen Befunde, so ergibt sich, dass dem Ausgange des Processes, der sich klinisch als Atrophie darstellt, ein lentescirender tropho-neurotischer Entzündungsprocess vorausgeht, der sich hauptsächlich in den oberen Cutislagen abspielt, einerseits zu Schwund und Atrophie des Papillarkörpers der Talg- und Schweissdrüsen und Haare führt, anderseits Hypertrophie und sclerose des neugebildeten Bindegewebes gleichwie des elastischen Gewebes der tieferen Cutis erzeugt, welche demgemäss beträchtlich verdickt und in ihrer Structur entsprechend verändert erscheint.

Die Veränderung manifestirt sich schon beim Excidiren eines Hautstïckchens durch das Kreischen des Gewebes beim Eindringen des Messers, und wie die mikroskopische Untersuchung erweist, durch das verschiedene Verhalten in Form und Farbe etc. der Gewcbselemente, indem die verschiedenen Cutislagen sich auch in verschiedener Weise auf die FarbstoffReagentien empfänglich zeigen u. a.

Die Rundzellenwucherung, die reichlichere Vascularisation und das gehäufte Vorkommen von Mastzellen weisen darauf hin, dass in den oberen Cutislagen eine chronische Entziindung sich abspiele, die endlich zur Atrophie führt.

Dem klinischen Bilde der Hautfelderung mit seinen erhabenen Leisten und etwas eingesunkenen weissen Flecken entsprechen histologisch, respective die vorgewölbten noch infiltrirters Partien und die bereits atrophischen Stellen.

Indem die grösstentheils stark verdünnte oberflächliche Lage einer dichten festen Unterlage aufruht und leicht iiber derselben verschoben werden kann (histologisch findet sich hier an der Grenzzone das elastische Gewebe am reichlichsten), kommt jenes eigenartige Bild zustande, dass die Haut "wie von einem gebratenen Apfel abgehoben werden kann" oder „zerknittertem Cigarettenpapier ähnlich sieht“. 
Mit dem Befund einer chronischen Entzündung der Haut steht auch das Gefühl beständigen Fröstelns des Kranken im Einklang.

Der relative Reichthum an glatten Muskelzellen und elastischem Gewebe ist wohl darauf zurückzuführen, dass durch theilweisen Untergang von Bindegewebe jene Gebilde prävaliren.

Wir halten es für unzulässig, über die Natur des Processes mehr zu behaupten, da ein Substrat hiefür nicht vorhanden ist. Wir haben auch keinen Grund, die Affection anders als "diffuse idiopathische Atrophie der Haut" zu bezeichnen. Es dünkt uns zur Zeit viel zweckmässiger, die Thatsachen genau festzustellen, beziehungsweise mitzutheilen, als - wie dies leider derzeit so oft geschieht - durch nene Namen Verwirrung zu erzeugen.

\section{Erklärung der Abbildungen auf Tafel XXVI u. XXVII.}

Tafel I. Ein Durchschnitt durch die Haut; Hämalaun-Eosin-Präparat. Zeiss Objectiv A, Ocular 2. 50fache Vergrösserung. a) Epidermislage, () oberflächliche, c) tiefere Cutisschichte, d) Panniculus adiposus, $e e)$ Schweissdrüsenknäuel, $f f$ ) quer- und längsgeschnittene Gefässe, g) Arrector pili, h) Haar mit kolbenartigen Auswüchsen der Wurzelseheiden, ii) längs und quer getroffene Nerven, $k$ ) Rest einer in dichten Bindegewebszügen eingeschlossenen Talgdrüse.

T afel II. Eine Stelle des Präparates bei $390 f a c h e r$ Vergrösserung. Zeiss Objectiv E, Ocular 2. Lithioncarminfärbung. a) Die in bandartigen Streifen abgehobene Hornschichte, a) Rete Malpighii mit gut entwickelter Stachelzellenschichte. Basalzellen zum Theil pigmentirt. b) Oberflächliche Cutislage, c) tiefere Cutisschichte in breiteren dichten Zügen und stark tingirt, cc) quer- und längsgeschnittene Gefässe, in deren Umgebung kleinzelliges Infiltrat, welches bei $g$ ) in das Rete Malpighii eingebrochen hat, $f$ ) Chromatophoren.

Tafel III. Färbung der elastischen Fasern mit saurem Orcein, vorgefärbt mit Lithionearmin.

Zeiss Objectiv C, Ocular 2, Vergr. 145. 
TAF. XXVI.

Fig.1.

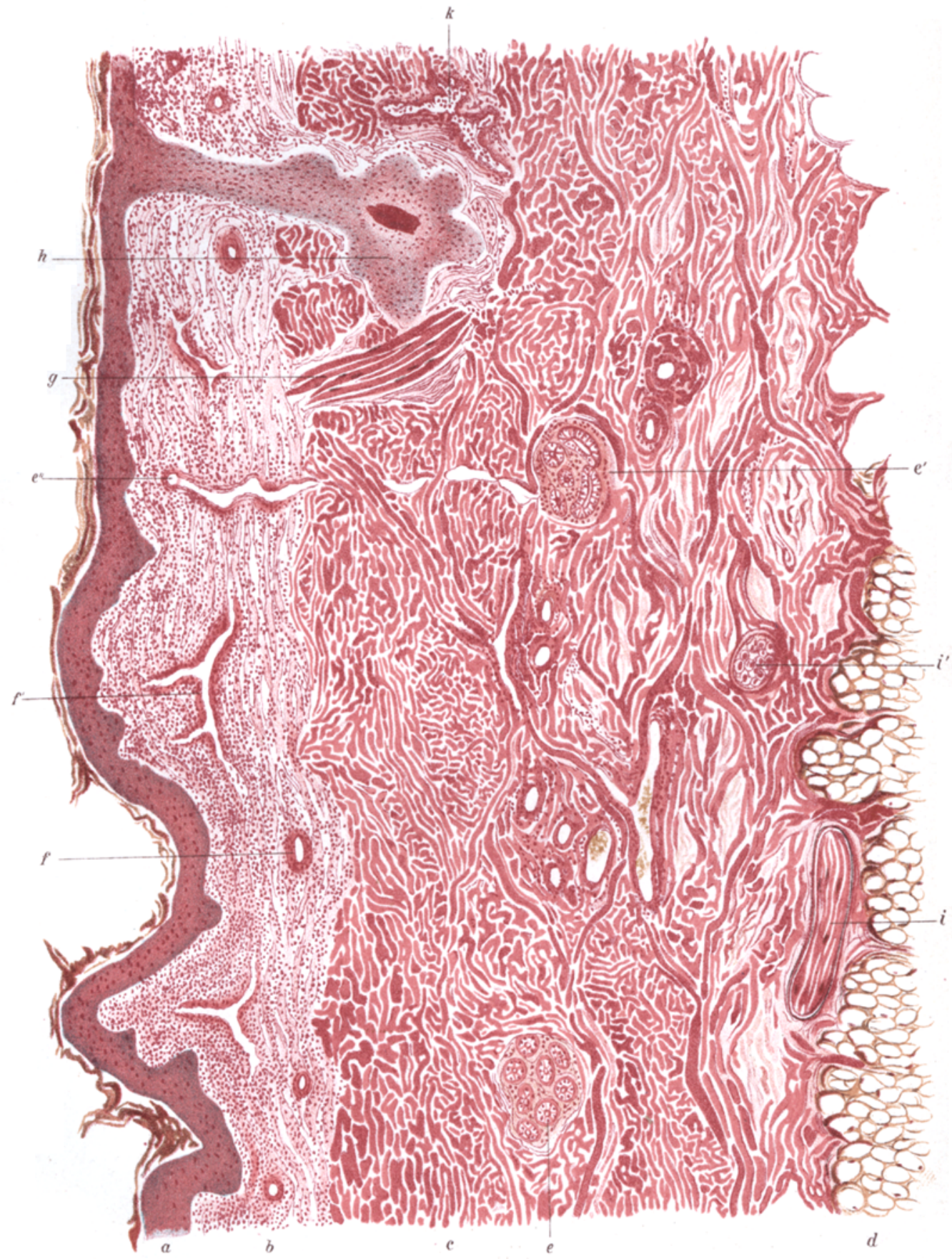

Neumann : Atrophie der Haut. 
Fig. 2.

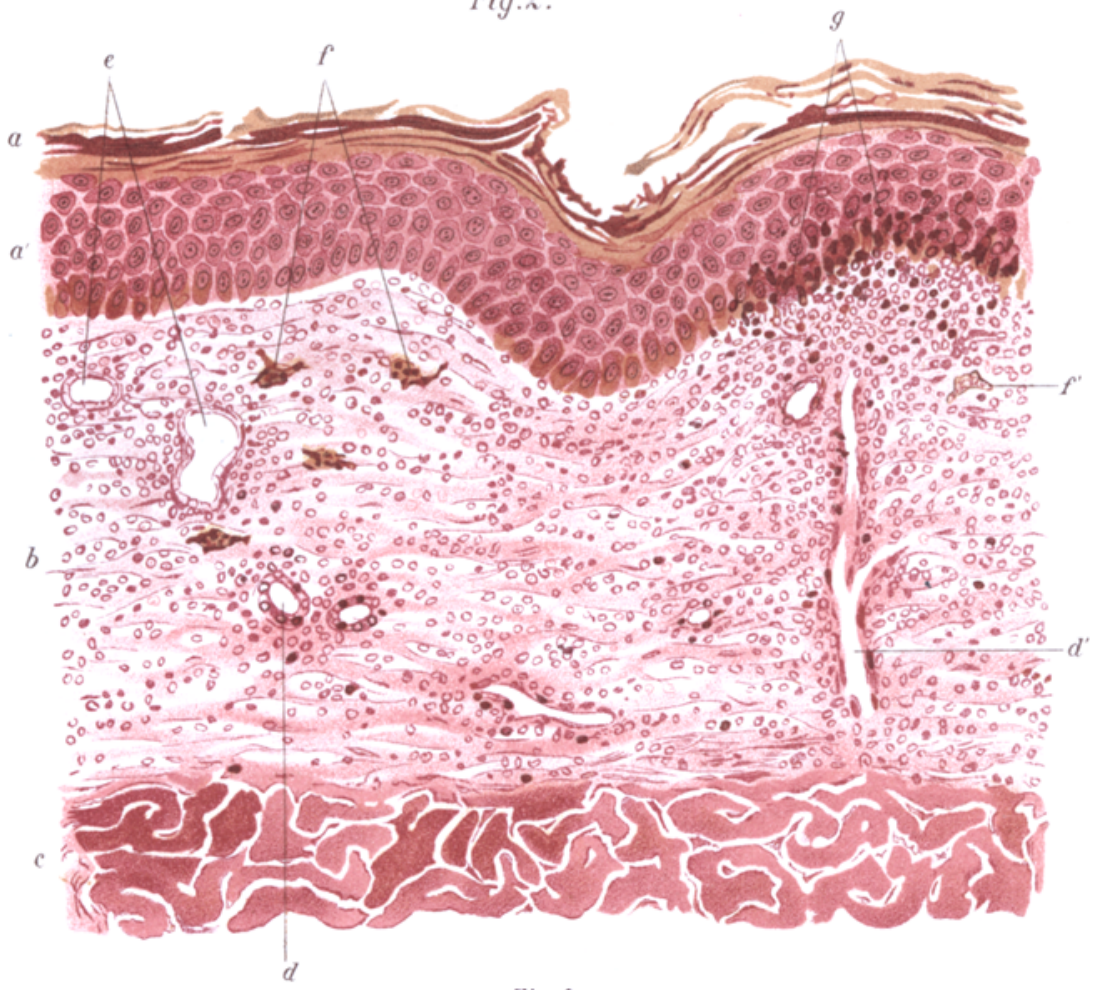

Fig.3.

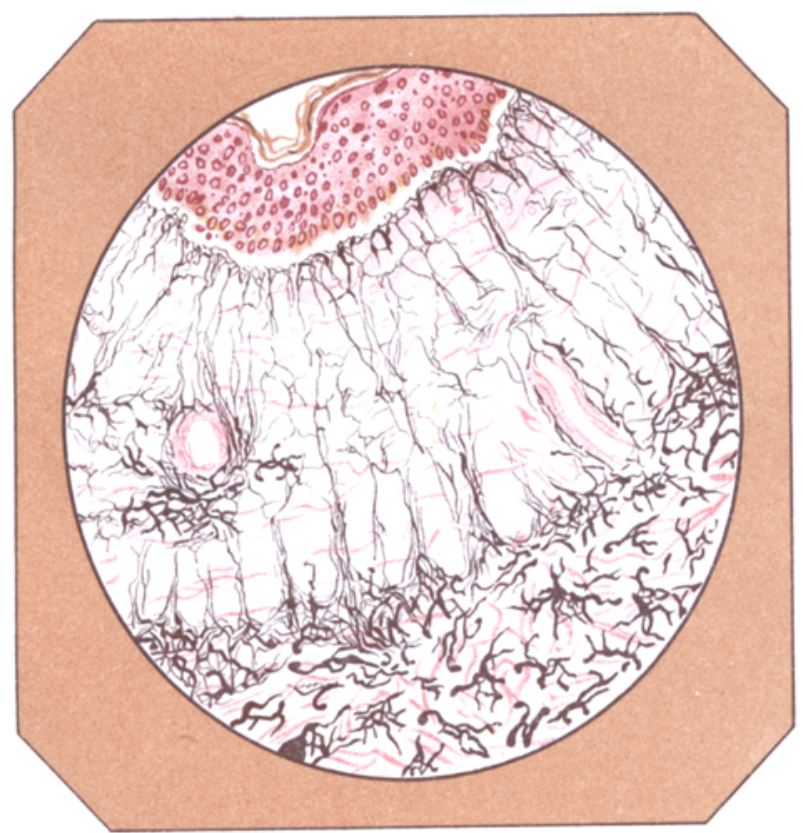

Neumann : Atrophie der Haut. 